\title{
Special issue on real-time processing of medical images
}

\author{
Mohamed Akil $^{1} \cdot$ Mohamed Hédi Bédoui $^{2}$
}

Published online: 16 March 2017

(c) Springer-Verlag Berlin Heidelberg 2017

This special issue on real-time processing of medical images addresses the current state-of-the-art in the field of parallel programming for medical imaging applications and the future trends in real-time medical image processing, including parallel computing (computational models, parallel algorithms and hierarchical memory optimizations), real-time implementation of embedded medical image processing applications on dedicated architectures and/or parallel architectures.

We are very grateful to the reviewers who provided valuable comments and suggestions to improve the quality of the papers accepted for this special issue.

Brief outlines of these papers are stated below:

The first paper by Yee Hui Lee, Mohamed Khalil-Hani, Rabia Bakhteri and Vishnu P. Nambiar presents a real-time image acquisition system with an improved image quality assessment module to acquire high quality near infrared images. The NIR image quality assessment module utilizes improved two-dimensional entropy and mask-based edge detection algorithms. In this work, the effectiveness of the proposed NIR image acquisition system is demonstrated through the implementation of a complete finger- vein biometric authentication system. The proposed module is implemented as an embedded system on a Field

Mohamed Akil

mohamed.akil@esiee.fr

Mohamed Hédi Bédoui

hedi.bedoui2015@gmail.com

1 ESIEE Paris, 2 BLD Blaise Pascal, 93162 Noisy-le-Grand, France

2 LTIM, Faculte de Medecine de monastir, Monastir, Tunisia, France
Programmable Gate Array (FPGA). The proposed system is able to achieve a recognition accuracy of $0.87 \%$ equal error rate (EER) and can handle real-time processing at 15 frames/second.

The second paper by Faten Chaieb, Tarek Ben Said, Sabra Mabrouk and Faouzi Ghorbel presents a segmentation method from four-phase computed tomography images. The proposed method is based on the combination of the Expectation-Maximization algorithm and the Hidden Markov Random Fields. The segmentation method is applied on a Volume Of Interest (VOI) that decreases the number of processed voxels. In order to accelerate the classification steps within the segmentation process, a bootstrap resampling scheme is applied. The proposed method is speeded up by adopting a bootstrap sampling approach on a semi-automatic selected VOI.

The third paper by Agnès Masson-Sibut and Amir Nakib presents a new framework of bone landmark extraction from ultrasound images on real-time. The proposed method takes into account both the ultra-sonic waves and the bones properties to obtain the best final result. The proposed method is stable and works for different patients.

The fourth paper by Nikolai Abtamov, Maxim Fradkin, Laurence Rouet and Hans-Aloys Wischmann describes a new hybrid image processing approach to block-matching that takes advantage of both types of algorithms: lossless and lossy. This proposed approach is adapted to the image content and noise, and provides high flexibility for the speed/accuracy trade-off. The proposed block-based motion estimation approach is scalable and dedicated for X-Ray and Ultrasound images.

The fifth paper by Mukul Shirvalkar, Yamuna Lagadapati and Xuanliang Dong describes the fast implementation of the semi-variogram algorithm and presents a technique for the fast computation of the semi-variogram using two 
FPGA architectures. A modular architecture approach is chosen to allow for replication of processing units. The algorithm is benchmarked using VHDL on a Xilinx XUPV5-LX11OT development kit.

The sixth paper by Adithya Upadhya, Basavaraj Talawar and Jeny Rajan presents the first successful attempt to implement a GPU accelerated version of NLML algorithm (Non Local Maximum Likelihood). The GPU NLML achieves a speedup of 5.12-6.24 for search window size from $10 \times 10 \times 10$ to $50 \times 50 \times 50$ voxels.

The seventh paper by Eduardo Alcain, Angel TorradoCarvajal, Antonio Sanz Montemayor and Norberto Malpica describes a fast patch-based algorithm and a performance study of this approach for the estimation of a certain image modality by using a multi-atlas and a 3D patch-based correlation approach. This paper also presents a parallel algorithm that synthesizes the new modality from a patchbased correlation approach in a 3D neighbourhood. Different hardware implementations (multithread CPU using OpenMP, GPU) and comparison are given.

The eighth paper by Jan Rühaak, Lars König, Florian Tramnitzke, Haral Köstler and Jan Modersitzki presents a generic approach to highly efficient image registration in two and three dimensions. Both mono-modal and multimodal problems are considered. The proposed method is implemented on multi-core CPU and GPU. With the proposed matric-free approach and code that uses the advantages offered by the GPU platform, it is possible to achieve registration results in just a few hundredths of a second for medium-sized medical images.

The ninth paper by Carlos Graca, Gabriel Falcao, Isabel N. Figueiredo and Samuel Kumar proposes on multi-GPU based parallel real-time algorithms for segmentation and shape-based object detection aims to accelerate two medical processing methods: automated blood detection in wireless capsule endoscopy images and automated bright lesion detection in retinal fundus images. The proposed multi-GPU framework for bright lesion detection in fundus images is able to process 62 frames per second with a average speedup of being 667 times faster than the equivalent CPU version. The accelerated method is running on multi-GPU systems for blood detection in wireless capsule endoscopy images that is on average 265 times faster than the original CPU version and is able to process 344 frames per second.

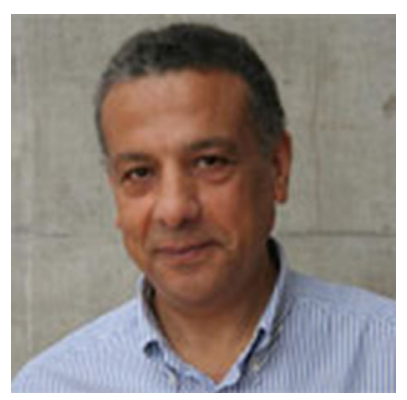

Mohamed Akil received his $\mathrm{Ph} . \mathrm{D}$. degree from Montpellier University (France) in 1981 and his doctorat d'état (DSc) from the Pierre et Marie curie University (UPMC, Paris, France) in 1985. Since September 1985, he has been with ESIEE Paris that is the CCIR's (Chambre de commerce et d'industrie de région Paris Ilede-France) center for scientific and engineering education and research. He is currently a Professor in the Computer Science Department, ESIEE Paris. He is a membrer of the Laboratoire d'Informatique Gaspard Monge, Université Paris-Est Marne-la-Valée (UMR 8049, unité mixte de recherche CNRS), a joint research laboratory between Université Paris-Est Marne-la-Vallée (UPEM), ESIEE Paris and École des Ponts ParisTech (ENPC). He is member of the program committee of the SPIE-Real Time Image and Video Processing conference (RTIVP). He is member of the Editorial Board of the Journal of Real-Time Processing (JRTIP). His research interests include dedicated and parallel architectures for image processing, image compression and virtual reality. His main research topics are parallel and dedicated architectures for real time image processing, reconfigurable architectures and FPGA, high-level design methodology for multi-FPGA, mixed architecture (DSP/FPGA) and Systems on Chip (SoC). He has published more than 160 research papers in the above areas.

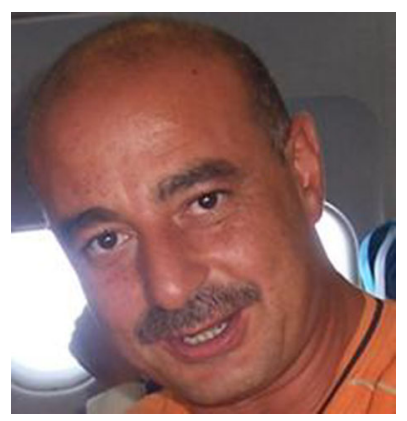

Mohamed Hédi Bédoui was born in 1965 in Teboulba, Tunisia. He is an Engineer in Electronics (ENIS-University of Sfax in 1988); He had his $\mathrm{Ph} . \mathrm{D}$. in Biomedical Engineering at the University of Lille, France, in 1993. He is full Professor of the TIM laboratory (Technologie et Imagerie Médicale, LTIM-Biophysics at the Faculty of Medicine of Monastir, Director of LR12ES06) and President of the Tunisian Association for the promotion of applied Research-ATUPRA. His current interests include modelisation and processing of medical data from the sensor to the optimization of embedded systems implementation. 